\title{
Grounding for EMC at the European XFEL
}

\author{
Herbert Kapitza, Hans-Jörg Eckoldt, Markus Faesing \\ Deutsches Elektronensynchrotron (DESY) \\ D-22603 Hamburg, Germany \\ Email: herbert.kapitza@desy.de
}

\begin{abstract}
The European X-Ray Free Electron Laser (XFEL) research facility is currently being built at Deutsches Elektronensynchrotron (DESY) in Hamburg, Germany. In this large underground facility high power RF stations are operated next to high precision diagnostics instruments. This made electromagnetic compatibility (EMC) an important issue from the very beginning of the project. A well-designed grounding system with a hierarchical structure is an essential ingredient for safety and EMC. Important parts of the facility ground were implemented in the course of the civil construction work. The specialties of tunnel boring and building far below the ground water level had to be taken into account. Some aspects of facility grounding were investigated at the free electron laser facility FLASH which operates for users at DESY for some years.
\end{abstract}

\section{THE EUROPEAN XFEL}

\section{A. General layout}

The European X-Ray Free Electron Laser (XFEL) research facility is currently being built at Deutsches Elektronensynchrotron (DESY) in Hamburg, Germany [1]. Fig. 1 shows a schematic overview of the XFEL. The facility comprises an injector (XTIN) with a laser-driven electron gun, a superconducting electron linear accelerator or linac (XTL), a fan of tunnels (XTD1...10) to which the accelerated electrons are distributed and where $\mathrm{X}$-ray photons are generated in undulators, and an experimental hall (XHEXP) where the photon experiments will be set up. Being located in the inhabited Hamburg area, most of the XFEL has been built underground, using two tunnel boring machines. The facility essentially is a set of buildings with up to seven floors below ground (shafts) and up to two floors above ground (halls), the shafts being connected by tunnels. The total length of the facility from the injector to the experimental hall is about $3.4 \mathrm{~km}$. The soil coverage varies from 10 to $30 \mathrm{~m}$ (Fig. 2).

\section{B. The superconducting electron linac}

The first two kilometers of the facility are occupied by the injector and the superconducting electron linac. The electron bunches are created by an optical laser pulsing a photo cathode. After passing a bunch shaping section the electrons are accelerated to an energy of $17.5 \mathrm{GeV}$. This allows to generate X-ray photons with wavelengths down to $0.1 \mathrm{~nm}$.

The XFEL main linac is a sequence of 25 more or less identical stations. Fig. 3 schematically shows such a station consisting of four accelerator modules. Each module is a cryogenic vessel which contains eight superconducting cavities made of niobium and operating in a superfluid helium bath at $T=2 \mathrm{~K}$. The cavities operate at a frequency of $1.3 \mathrm{GHz}$. For each station the required RF power is generated by a klystron and then brought to the cavities via waveguides and couplers.

The high voltage pulses for the klystron are generated by a power converter (modulator). The nominal modulator pulse data are $V=8.9 \mathrm{kV}, I=1.5 \mathrm{kA}, t=1.57 \mathrm{~ms}$ at a repetition rate of $10 \mathrm{~Hz}$. A 1:12 pulse transformer next to the klystron converts these pulses to the klystron level: $V=105 \mathrm{kV}, I=125 \mathrm{~A}$. Klystrons and pulse transformers are located inside the tunnel, close to the accelerator modules, while the modulators are installed in the hall XHM above ground (Fig. 1). The pulse transformers are connected to the modulators via shielded coaxial pulse cables which are up to $1.5 \mathrm{~km}$ long. This topology allows for modulator maintenance during XFEL operation. Installing the modulators close to the klystrons - as is the case for many accelerators - would have required expensive additional underground constructions. The pulse cables will be laid on the center trays in the compartment underneath the tunnel floor (Fig. 3).

For $25 \mathrm{RF}$ stations in the main linac and two in the injector the modulator pulse data result in a maximum pulse power of $360 \mathrm{MW}$ being sent into the tunnel. Even if only on the per mill level, current leakage from the high power pulse cables might considerably interfere with the operation of nearby instruments for beam diagnostics, femtosecond synchronization systems, and other sensitive equipment. Under such circumstances it was considered important to address questions of electromagnetic compatibility (EMC) already in the early design phase of the XFEL project.

\section{XFEL FACILITY GROUNDING}

\section{A. General structure}

An important ingredient for safety and EMC is a welldesigned grounding system. The main purpose of a grounding system is to offer noise currents a low impedance return path to their sources, thus shunting them away from delicate parts of the installation. In an extended facility like the XFEL the grounding system must be hierarchically organized. Then local grounding systems connect to a facility ground mesh which includes as much as possible of the metal infrastructure of the facility. Besides cable trays and pipes, a sizable fraction of that metal is the reinforcement steel in the shaft and tunnel walls.

Local grounding naturally corresponds to the topology of the facility. In the shaft and hall buildings single floors, in some cases even single rooms form local systems. In the linac 


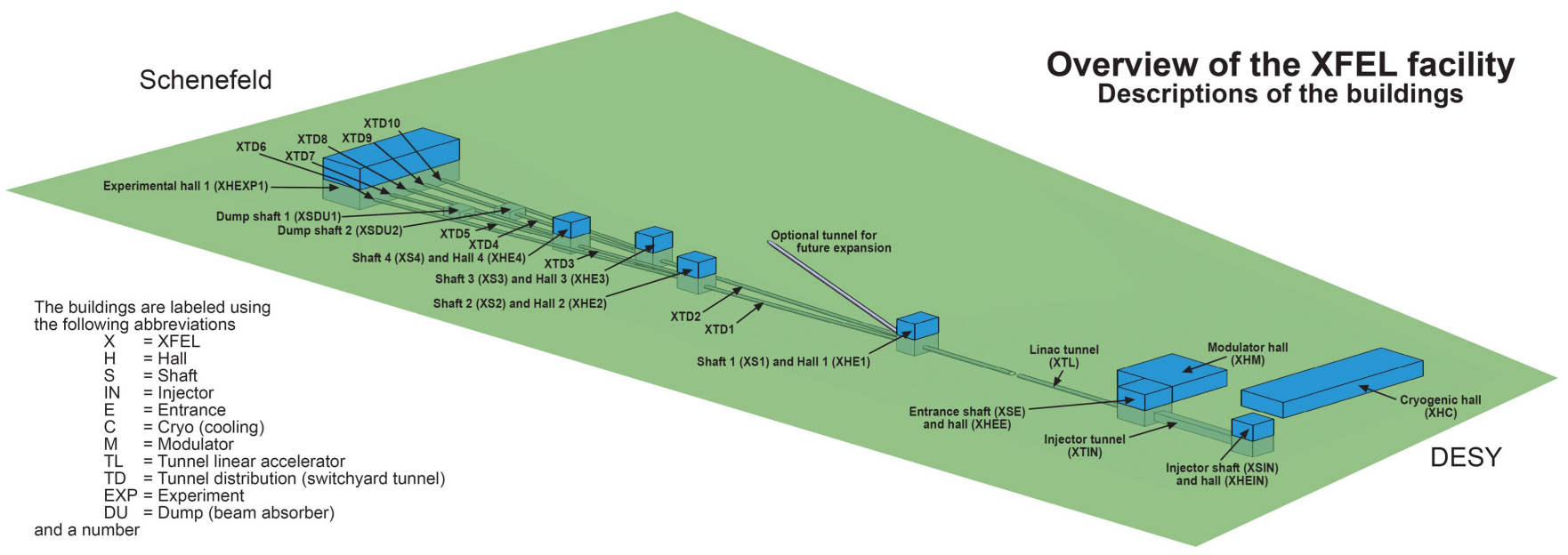

Fig. 1. General layout of the European XFEL.

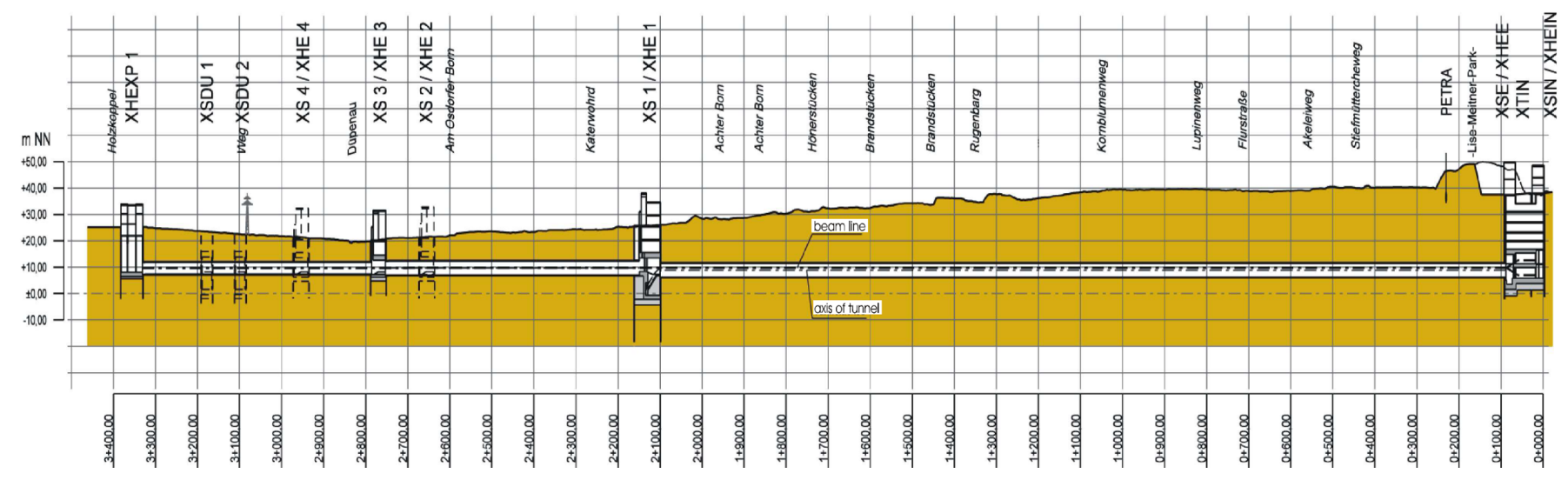

Fig. 2. Side view of the European XFEL.

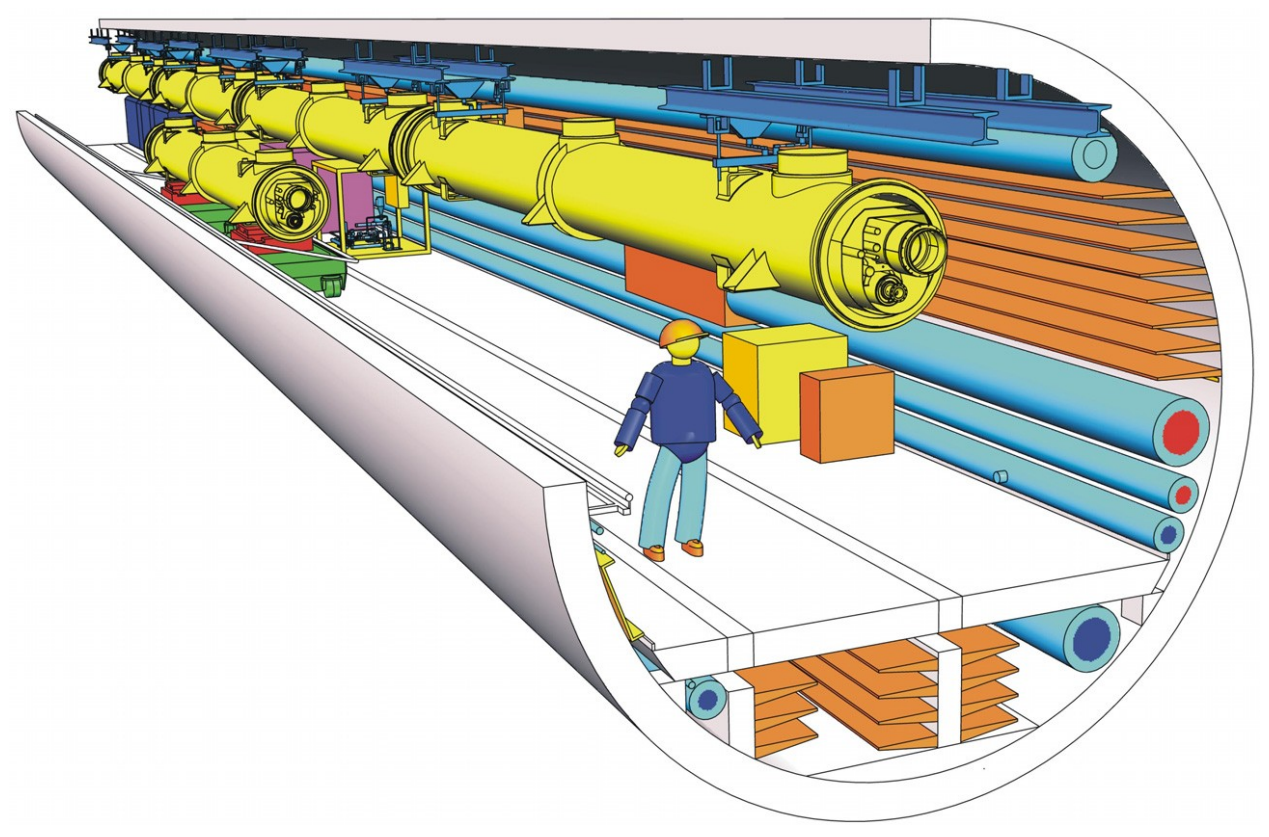

Fig. 3. Linear accelerator section of the European XFEL. 


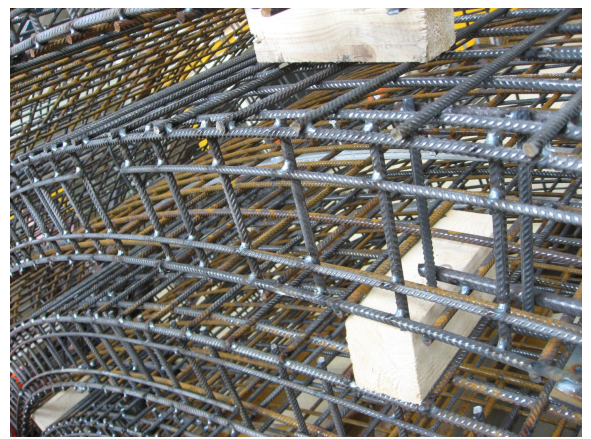

(a) Steel reinforcement cages of lining segments.

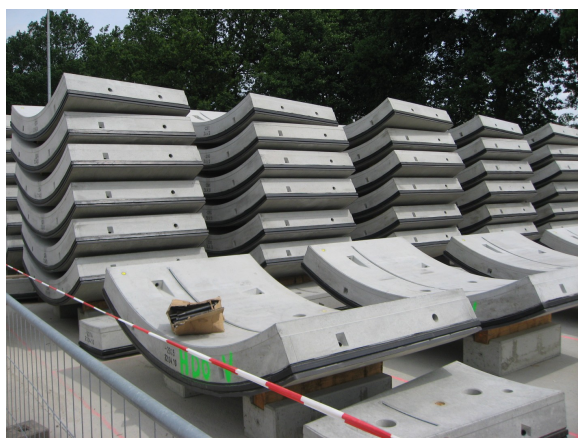

(d) Produced lining segments.

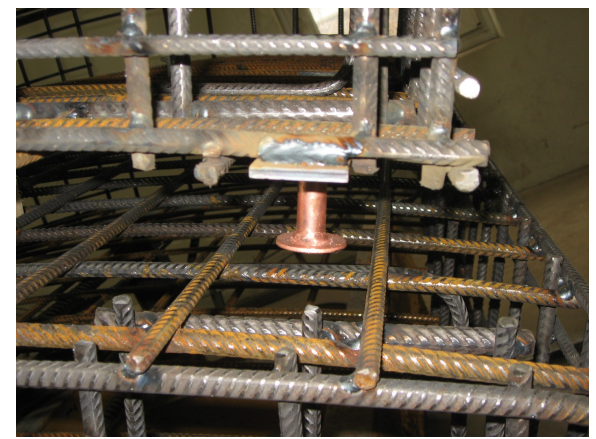

(b) Ground connector welded to steel cage.

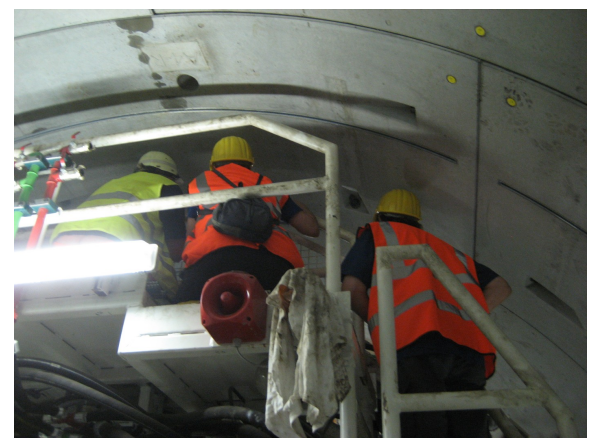

(e) Lining segments put in place.

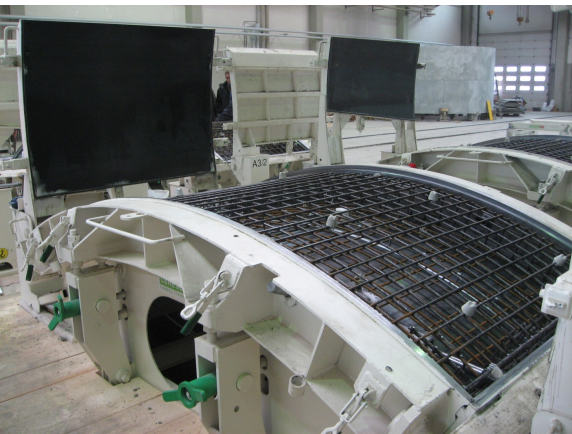

(c) Steel cage in a lining segment mould.

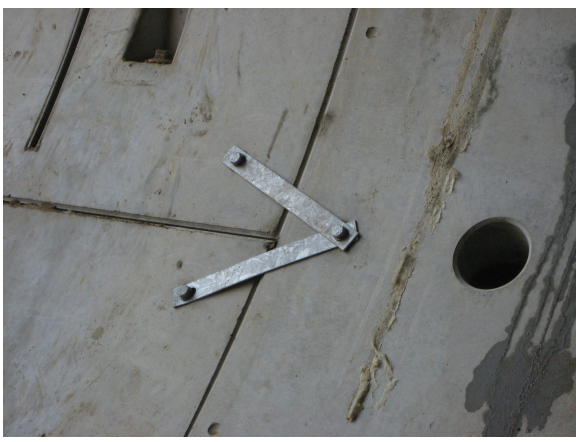

(f) Electrically connected lining segments.

Fig. 4. Inclusion of the tunnel wall steel in the XFEL facility ground.

tunnel local systems are defined by the RF stations mentioned above.

\section{B. XFEL tunnel grounding}

Using a tunnel boring machine for building a tunnel results in a tunnel wall which is composed of relatively small pieces, so-called lining segments. This complicates the inclusion of the steel of the tunnel wall in the facility ground. In the XFEL each tunnel is a sequence of $1.5 \mathrm{~m}$ long rings, each ring consisting of six lining segments plus a small keystone. The segment dimensions are $1.5 \mathrm{~m}$ along the tunnel axis and $3 \mathrm{~m}$ along its circumference. In total about 23000 lining segments were placed in the XFEL tunnel walls.

The picture sequence in Fig. 4 shows various details of including the steel of the tunnel wall in the XFEL facility ground. Each lining segment contains a massively welded steel reinforcement cage (Fig. 4a). In order to make it accessible from outside, three threaded ground connectors are welded to it (Fig. 4b). The prepared cage - with protected ground connector threads - is put in a mould (Fig. 4c) which is then filled with concrete. In this way 70 lining segments were produced per day (Fig. 4d). After placement by the tunnel boring machine (Fig. 4e) each lining segment was electrically connected to all its neighbours using screws and steel strips (Fig. 4f). In this way all XFEL tunnels were made conducting tubes. Using the $\lambda / 50$-rule, the grounding grid mesh size given by the size of the lining segments - approximates a ground plane for noise current frequencies up to a few MHz.
Each lining segment has an integrated anchor rail for fixing pipes, cable trays and other infrastructure. These rails are also welded internally to the steel reinforcement cage. In this way all metal infrastructure mounted on the anchor rails is automatically included in the XFEL facility ground. In addition in every tunnel there is a dedicated continuous grounding rail made of $40 \times 5 \mathrm{~mm}^{2}$ steel band. Besides being required by law, this also facilitates the bonding of local equipment.

The complicated floor structure shown in Fig. 3 has been implemented using pre-cast concrete parts for the cable compartments and the floor slabs. Their steel reinforcement is made accessible in a similar manner as described above for the tunnel segments. The floor elements are connected to the tunnel wall so that in particular the pulse cable trays are automatically included in the XFEL facility ground.

\section{XFEL shaft and hall grounding and lightning protection}

One peculiarity of building the European XFEL was the high ground water level on the construction sites, some shafts reaching $12 \mathrm{~m}$ deep into the ground water. The shaft buildings were built up in pits made of slurry walls and underwater concrete plates. The slurry walls were constructed by digging up to $40 \mathrm{~m}$ deep and $1.5 \mathrm{~m}$ wide slits in the ground, inserting reinforcement steel cages, and finally pouring in the concrete. Once the pit walls were completed, the soil was excavated from the inside, the last meters of excavation taking place underwater. Then the floor of the pit was installed as an underwater concrete plate. After pumping out the water, the 


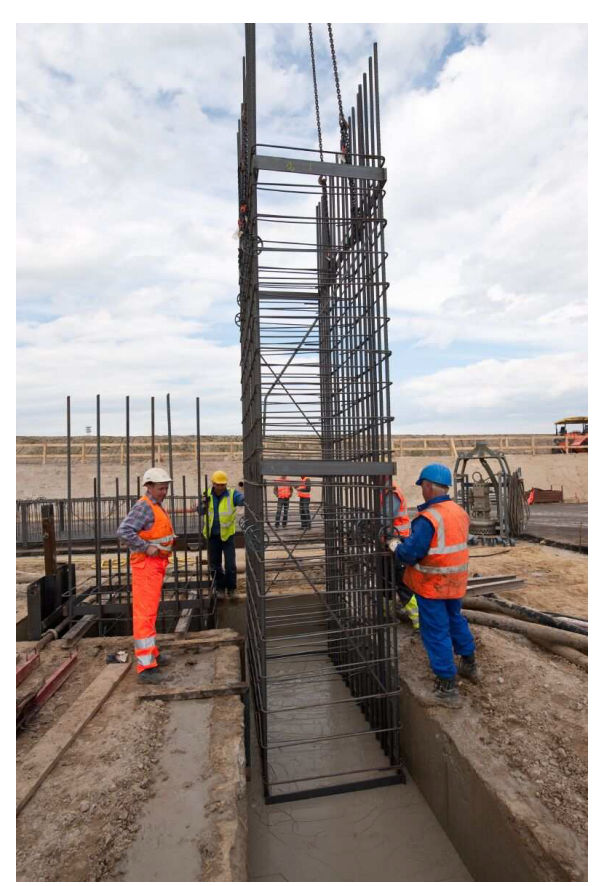

(a) Insertion of a reinforcement steel cage into a slit for a slurry wall.

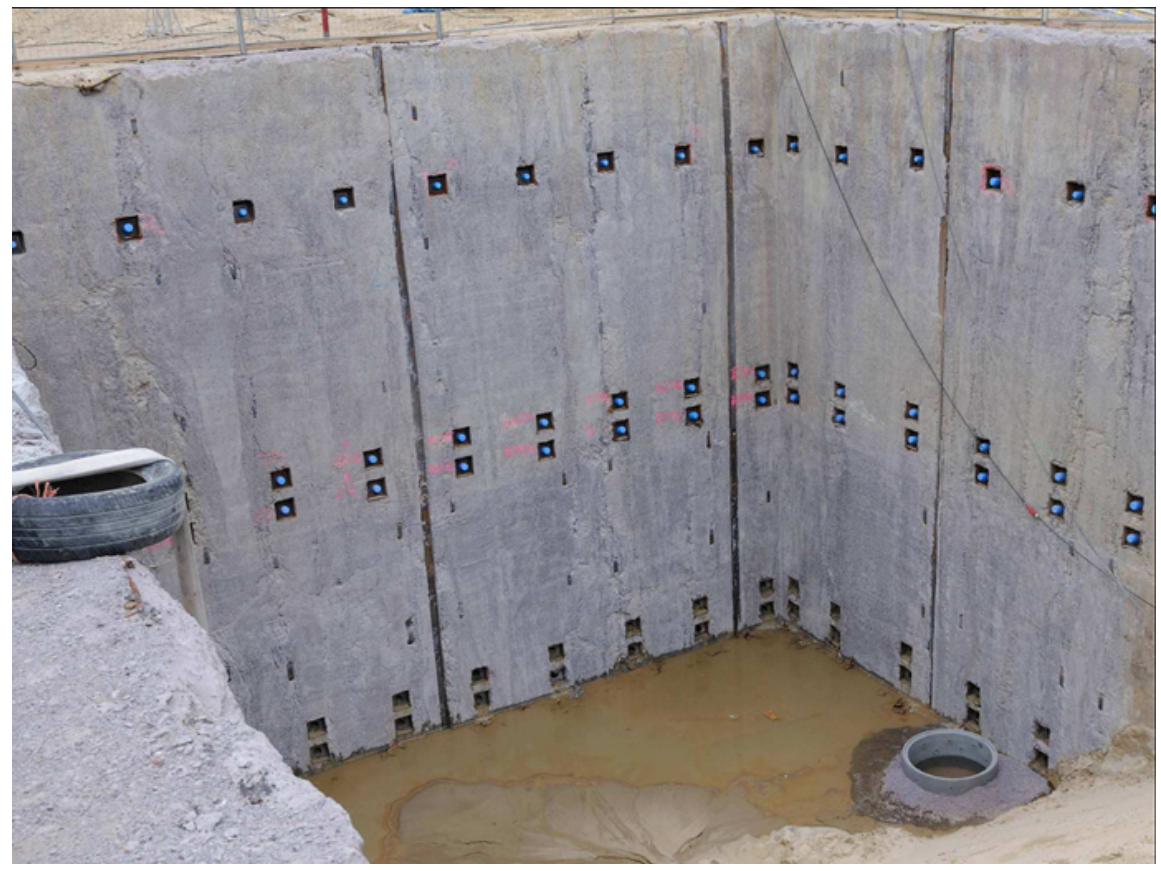

(b) Anchored slurry walls of a $30 \mathrm{~m}$ deep building pit.

Fig. 5. Construction details of a shaft building pit.

pit was ready for building up the shaft building. After finishing the building the remaining pit was refilled with sand.

Fig. 5 shows some details of the pit construction. The pit walls and the floor are anchored in the surrounding soil to prevent floatation and collapse from earth and water pressure. The metal anchors as well as the reinforcement steel in the pit walls make very good contact to the earth and were incorporated in the lightning protection system of the hall building above ground.

The grounding of the XFEL shaft and hall buildings followed conventional civil construction procedures. Some details are schematically shown in Fig. 6:

- The foundation earth electrode (5) between the pit floor plate (2) and the shaft base plate (4) is connected (6) to the anchored slurry walls (1) which provide good earth contact. This dedicated earth electrode is a grid of stainless steel rods with a mesh size of $10 \times 10 \mathrm{~m}^{2}$. It is needed because the shaft walls $(3)$ and floors $(4,11)$ are made of watertight concrete which prevents good earth contact of any contained metal.

- Since most of the reinforcement steel in the shaft walls and floors is not welded but wire-tied, dedicated grounding grids with a $5 \times 5 \mathrm{~m}^{2}$ mesh size are incorporated there. In some critical regions half the mesh size is used. Adapted to the room topology in the building, external grounding points are provided, usually combined with equipotential bonding (7-10, 14-20).

- At a tunnel entrance to a shaft both building grounds are connected at four points around the tunnel circumference
(13). Another important building ground connection are the vertical pulse cable trays from the modulator hall XHM down to the entrance shaft XSE.

- The lightning protection system conforms to Protection Class III of the norm EN IEC 62305 which requires a $15 \times 15 \mathrm{~m}^{2}$ lightning conductor (21) mesh size. It includes lightning rods (22) to protect electrically connected roof equipment (23). A ring earth electrode (20) around the hall building serves potential steering.

\section{Pulse cable tests at Flash}

FLASH is a free electron laser facility at DESY which operates in user mode since 2005 . FLASH is only $300 \mathrm{~m}$ long, but many XFEL design features, in particular the superconducting accelerator modules, evolved from it.

From the very beginning of the XFEL planning, the pulse cables from the modulators above ground to the pulse transformers in the tunnel were a major point of EMC concern. Therefore a test was performed where a $1.5 \mathrm{~km}$ long bundle of four pulse cables was laid across the DESY site from a distant modulator to a pulse transformer of the FLASH facility. The cable bundle was routed intentionally through the FLASH tunnel, parallel to the accelerator.

At FLASH the reinforcement steel of the tunnel wall is not a part of the facility ground, only metal pipes, cable trays, and a grounding rail are included. Failure in one of these components has immediate consequences elsewhere:

- Initially the first $12 \mathrm{~m}$ of the pulse cable bundle at the entrance to the FLASH tunnel were installed without a supporting cable tray. Common mode current spikes of 


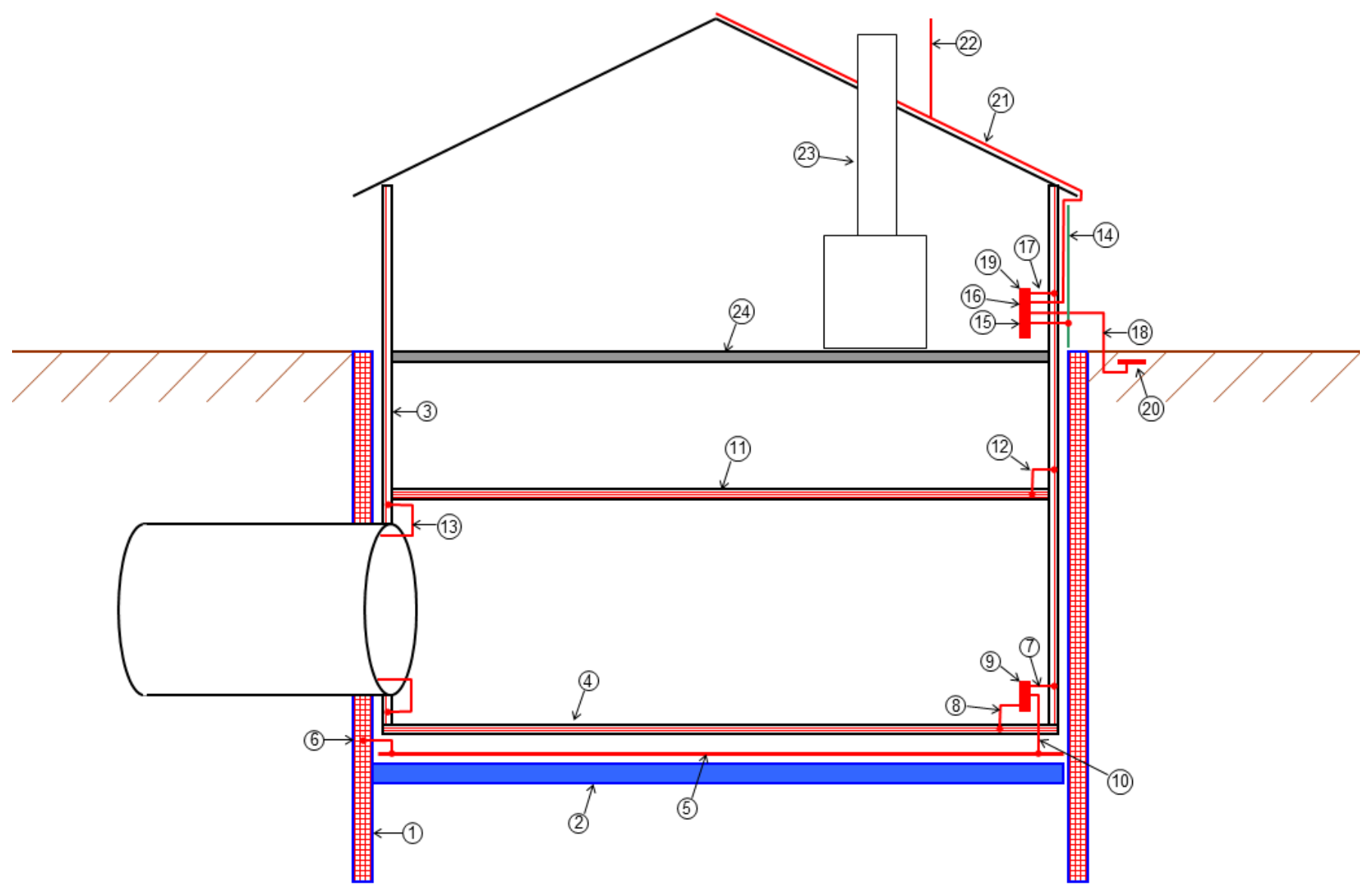

Fig. 6. Grounding and lightning protection scheme for the XFEL halls and shafts.

2.5 A were measured in the pulse cable bundle at the start and at the end of the modulator pulse. This common mode current returned in the environment, e.g. $0.5 \mathrm{~A}$ in the beam tube of the accelerator.

- After installing a provisional cable tray by wrapping the cable bundle in copper foil and connecting to the adjacent cable trays the common mode current spikes reduced to $0.2 \mathrm{~A}$.

- In the regions of sensitive FLASH instrumentation the pulse cable bundle was from the beginning properly installed on cable trays or metal walkways. Therefore neither the operators of the FLASH accelerator nor the users of the FEL radiation experienced any performance degradation with the pulse cables in operation.

This test demonstrated how a good grounding system absorbs a sizable fraction of the noise current from an installation. It also showed that with proper grounding the pulse cables can be operated close to an accelerator without causing EMC problems.

\section{CONCLUSION}

The European XFEL research facility is currently being built at DESY in Hamburg, Germany. In this large underground facility X-ray laser radiation will be created by sending accelerated electrons through undulators. In the accelerator tunnel high power RF stations will be operated next to sensitive diagnostics equipment. In order to support EMC among so different systems, a solid hierarchical grounding scheme has been developed for the XFEL. Large parts of the facility ground were implemented in the course of the civil construction work. Here the peculiarities of tunnel boring and building far below the ground water level had to be considered. The up to $1.5 \mathrm{~km}$ long pulse cables, which will send high voltage pulses from the modulator hall down into the accelerator tunnel, were tested at the FLASH accelerator at DESY. Experience from these tests entered the XFEL grounding discussion.

While the civil construction work is still going on, the technical installation of the European XFEL has started. First operation of the research facility is expected for 2015 .

\section{REFERENCES}

[1] M. Altarelli et al. (Editors), The European X-Ray Free-Electron Laser Technical Design Report, DESY Report 2006-097, Hamburg, July 2007. Also available via: http://www.xfel.eu/documents/technical_documents/. 